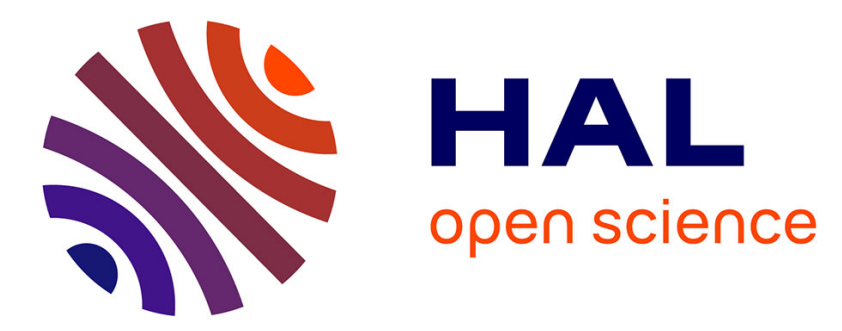

\title{
A relaxed Lyapunov-Krasovskii condition for global exponential stability of Lipschitz time-delay systems
}

Antoine Chaillet, Jakub Orlowski, Pierdomenico Pepe

\section{To cite this version:}

Antoine Chaillet, Jakub Orlowski, Pierdomenico Pepe. A relaxed Lyapunov-Krasovskii condition for global exponential stability of Lipschitz time-delay systems. 58th IEEE Conference on Decision and Control (CDC), Dec 2019, Nice, France. hal-03114574

\section{HAL Id: hal-03114574 \\ https://hal-centralesupelec.archives-ouvertes.fr/hal-03114574}

Submitted on 19 Jan 2021

HAL is a multi-disciplinary open access archive for the deposit and dissemination of scientific research documents, whether they are published or not. The documents may come from teaching and research institutions in France or abroad, or from public or private research centers.
L'archive ouverte pluridisciplinaire HAL, est destinée au dépôt et à la diffusion de documents scientifiques de niveau recherche, publiés ou non, émanant des établissements d'enseignement et de recherche français ou étrangers, des laboratoires publics ou privés. 


\title{
A relaxed Lyapunov-Krasovskii condition for global exponential stability of Lipschitz time-delay systems
}

\author{
Antoine Chaillet, Jakub Orłowski, and Pierdomenico Pepe
}

\begin{abstract}
For nonlinear time-delay systems with globally Lipschitz vector fields, we propose a relaxed sufficient condition for global exponential stability (GES), in which the dissipation rate of the Lyapunov-Krasovskii functional is not needed to involve the functional itself, but merely the point-wise current value of the solution. Our proof technique consists in explicitly constructing a Lyapunov-Krasovskii functional that satisfies existing criteria for GES. Consequences for robustness to exogenous inputs are briefly evoked and an example taken from neuroscience literature illustrates the applicability of the result.
\end{abstract}

\section{INTRODUCTION}

A powerful tool to study the stability of nonlinear timedelay systems is the Lyapunov-Krasovkii approach. This method requires to find a proper functional that decreases along the solutions of the system [10], [5], [8], [14]. The key difference with the Lyapunov approach for delay-free systems is that the object of study is no longer a function, but rather a functional (as its argument is the whole state history over a bounded time interval).

In order to ensure asymptotic stability, the LyapunovKrasovskii approach requires that the derivative of the functional $V$ dissipates in a point-wise manner, in the sense that its derivative along solutions needs to be negative as long as $x(t) \neq 0$. This light requirement turns out to be very handy in practice. However, when it comes to exponential stability, the only existing results require a dissipation rate that involves the whole functional itself (of the form $\dot{V} \leq-\varepsilon V$, see [10], [18], [22]): we call this a strict dissipation. In a delay free context, strict and point-wise dissipations are the same, as the Lyapunov function is upper and lower bounded by the state norm. However, for time-delay systems, these two bounds often differ (the lower bound involves the point-wise value of the solution's norm, whereas the upper bound involves the supremum norm of the state history).

The question of what stability properties can be guaranteed with a point-wise dissipation was already the subject of [3] and [2] for input-to-state stability properties and some questions remain open in that respect. Here, we address systems without input and show that a point-wise dissipation is indeed enough to show global exponential stability for systems with globally Lipschitz dynamics. Our proof is constructive: based

A. Chaillet is with Laboratoire des Signaux et Systèmes, CentraleSupélec, Univ. Paris Saclay, Gif sur Yvette, France and with Institut Universitaire de France antoine.chaillet@centralesupelec.fr. J. Orłowski is with Laboratoire des Signaux et Systèmes, CentraleSupélec, Univ. Paris Saclay, Gif sur Yvette, France jakub. orlowski@l2s. centralesupelec.fr.

P. Pepe is with University of L'Aquila, Italy pierdomenico.pepedunivaq.it on a functional with point-wise dissipation, we explicitly construct a functional that dissipates in terms of the whole state history norm. For globally Lipschitz dynamics, we show that point-wise, strict, and history-wise dissipations are the same from the point of view of global exponential stability.

We briefly describe how this result can be used to study robustness to exogenous inputs. We also illustrate its applicability with an example taken from neuroscience literature.

\section{Problem Statement}

We consider nonlinear time-delay systems of the form:

$$
\dot{x}(t)=f\left(x_{t}\right) .
$$

The state $x_{t}$ is defined as $x_{t}(s):=x(t+s)$ for all $s \in[-\delta ; 0]$, where $\delta \geq 0$ denotes the maximum delay of the system. For each $t \geq 0, x_{t} \in \mathcal{C}^{n}$ where $\mathcal{C}:=C([-\delta ; 0], \mathbb{R})$, the set of continuous functions from $[-\delta ; 0]$ to $\mathbb{R}$. The vector field $f: \mathcal{C}^{n} \rightarrow \mathbb{R}^{n}$ is assumed to be Lipschitz on bounded sets (it will actually be assumed globally Lipschitz in most of the paper, but the introductory results below do not require this) and to satisfy $f(0)=0$.

We make use of two different norms. Given a vector $z \in \mathbb{R}^{n},|z|$ denotes its Euclidean norm, namely: $|z|:=\sqrt{\sum_{i=1}^{n} z_{i}^{2}}$. Given a function $\phi \in \mathcal{C}^{n},\|\phi\|:=$ $\max _{s \in[-\delta ; 0]}|\phi(s)|$. We will also make use of comparison functions: $\alpha: \mathbb{R}_{\geq 0} \rightarrow \mathbb{R}_{\geq 0}$ is of class $\mathcal{K}$ if it is zero at zero, continuous, and increasing; $\alpha \in \mathcal{K}_{\infty}$ if $\alpha \in \mathcal{K}$ and it is unbounded; $\beta \in \mathcal{K} \mathcal{L}$ if, for each $t \geq 0, \beta(\cdot, t) \in \mathcal{K}$ and, for each $s \geq 0, \beta(s, \cdot)$ is continuous, non-increasing, and tends to zero as its argument tends to infinity.

A sufficient condition for global asymptotic stability of (1) dates back to the fifties [10]. It makes use of LyapunovKrasovskii functionals (LKF), which have become a classical tool for the stability analysis of time-delay systems. It states that, if there exist a functional $V: \mathcal{C}^{n} \rightarrow \mathbb{R}_{\geq 0}$ and $\mathcal{K}_{\infty}$ functions $\underline{\alpha}, \bar{\alpha}$, and $\alpha$ such that, for all $\phi \in \mathcal{C}^{n}$,

$$
\begin{array}{r}
\alpha(|\phi(0)|) \leq V(\phi) \leq \bar{\alpha}(\|\phi\|) \\
D_{\text {囬 }}^{+} V(\phi) \leq-\alpha(|\phi(0)|),
\end{array}
$$

then the origin of (1) is globally asymptotically stable. In this result, formally stated in e.g. [5, Theorem 2.1, p. 105], $D_{\text {血 }}^{+} V$ denotes the upper-right Dini derivative of $V$ along the solutions of (1), namely:

$$
D_{\text {责 }}^{+} V(\phi):=\limsup _{h \rightarrow 0^{+}} \frac{V\left(x_{h}(\phi)\right)-V(\phi)}{h} .
$$

Several aspects are worth noticing. First, the upper and lower bounds on the LKF $V$ differ: the upper bound involves the 
whole norm $\|\phi\|$ of the state history whereas the lower bound involves solely the current value of the solution's norm $|\phi(0)|$. Using the terminology of e.g. [12], $V$ does not need to be coercive (which would require a lower bound involving the whole $\|\phi\|)$. This turns out to be useful in some applications. More crucially, the Dini derivative of $V$ is required to dissipate only in terms of the current value of the solution's norm, rather than the LKF itself. This key property, which we name "point-wise dissipation", often simplifies the analysis, as will be illustrated in Section V A particular form of global asymptotic stability is the following.

Definition 1 (GES) The origin of system (1) is globally exponentially stable (GES) if there exists $\eta, \gamma>0$ such that, for each $x_{0} \in \mathcal{C}^{n}$, its solution satisfies

$$
\left|x\left(t ; x_{0}\right)\right| \leq \eta\left\|x_{0}\right\| e^{-\gamma t}, \quad \forall t \geq 0 .
$$

GES is a powerful stability property, as it ensures both an exponential decay to the origin and a transient overshoot proportional to the initial state norm $\left\|x_{0}\right\|$. Moreover, under regularity conditions on the vector field, this property is known to ensure robustness to exogenous disturbances in an input-to-state stability (ISS) sense [22].

LKF characterizations of exponential stability also exist for a long time. Nevertheless, quite surprisingly, none of them allow for a point-wise dissipation rate. In order to make this more precise, we introduce the following terminology.

Definition 2 (GES LKF) Let $V: \mathcal{C}^{n} \rightarrow \mathbb{R}_{\geq 0}$ be a functional, Lipschitz on bounded sets of $\mathcal{C}^{n}$, for which there exist $\underline{k}, \bar{k}>0$ such that, for all $\phi \in \mathcal{C}^{n}$,

$$
\underline{k}|\phi(0)|^{2} \leq V(\phi) \leq \bar{k}\|\phi\|^{2} .
$$

Then $V$ is said to be:

- $a$ GES LKF with history-wise dissipation for (1) if there exists $k>0$ such that

$$
D_{\text {囬 }}^{+} V(\phi) \leq-k\|\phi\|^{2}, \quad \forall \phi \in \mathcal{C}^{n},
$$

- $a$ strict GES LKF for (1) if there exists $k>0$ such that

$$
D_{\text {[1] }}^{+} V(\phi) \leq-k V(\phi), \quad \forall \phi \in \mathcal{C}^{n},
$$

- $a$ GES LKF with point-wise dissipation for 11 if there exists $k>0$ such that

$$
D_{\text {[1] }}^{+} V(\phi) \leq-k|\phi(0)|^{2}, \quad \forall \phi \in \mathcal{C}^{n} .
$$

These three types of GES LKF thus differ only in the way they dissipate along the solutions of (1). Clearly, any GES LKF with history-wise dissipation is a strict GES LKF, which is also a GES LKF with point-wise dissipation.

The fact that GES can be established with a strict LKF (hence, with an LFK with history-wise dissipation) is known [10], [18], [22]. However, obtaining a strict LKF on practical examples usually requires some tricks. For instance, for an LKF that involves only the sum of terms of the form $\left|\phi_{i}(0)\right|^{2}$ and $\int_{-\delta}^{0}\left|\phi_{i}(s)\right|^{2} d s$ (as is often the case), we can add a term in the kernel of the integral (e.g. exponential) to get a dissipation involving the whole LKF [17], [7], [11].

Still, these tricks are not guaranteed to work with any LKF. Moreover, the use of GES LKF with point-wise dissipation would be much handier in practice and would homogenize GES theory with that for global asymptotic stability. Our main result establishes that these three notions of LKF are actually equivalent for systems defined by a globally Lipschitz vector field and ensure global exponential stability.

\section{MAIN RESULT}

Our main result, proved in Section IV is the following.

Theorem 1 (GES characterizations) Let $f: \mathcal{C}^{n} \rightarrow \mathbb{R}^{n}$ be globally Lipschitz and satisfy $f(0)=0$. Then the following statements are equivalent:

i) (1) admits a GES LKF with history-wise dissipation

ii) (1) admits a strict GES LKF

iii) (1) admits a GES LKF with point-wise dissipation

iv) the origin of (1) is GES.

For globally Lipschitz systems, point-wise dissipation is thus sufficient to establish GES and the existence of a strict GES LKF, and even a GES LKF with history-wise dissipation, then come for free.

The above result can prove useful when studying robustness to exogenous disturbances. For this, the main tool is ISS, which was originally introduced for delay-free systems [19] and then extended to time-delay systems [21], [17], [9]. It concerns systems of the form:

$$
\dot{x}(t)=f\left(x_{t}, u(t)\right),
$$

where $f: \mathcal{C}^{n} \times \mathbb{R}^{m} \rightarrow \mathbb{R}^{n}$ is Lipschitz on bounded sets and satisfies $f(0,0)=0$. The input $u$ is assumed to be in $\mathcal{U}^{m}$, the set of signals $u: \mathbb{R}_{\geq 0} \rightarrow \mathbb{R}^{m}$ that are measurable and locally bounded. We recall the definition of ISS below.

Definition 3 (ISS) The system (2) is input-to-state stable (ISS) if there exist $\beta \in \mathcal{K} \mathcal{L}$ and $\mu \in \mathcal{K}_{\infty}$ such that, for any $x_{0} \in \mathcal{C}^{n}$ and any $u \in \mathcal{U}^{m}$, its solution satisfies

$$
\left|x\left(t ; x_{0}, u\right)\right| \leq \beta\left(\left\|x_{0}\right\|, t\right)+\mu\left(\sup _{s \in[0 ; t]}|u(s)|\right), \quad \forall t \geq 0 .
$$

We refer the reader to the survey [20] for more information about ISS. It was shown in [22] that GES guarantees ISS under some regularity assumptions on $f$. Thus, the following is a direct consequence of Theorem 1 .

Corollary 1 (GES \& ISS) Assume the input-free system $\dot{x}(t)=f\left(x_{t}, 0\right)$ admits a GES LKF with point-wise dissipation. Assume further that $f(\cdot, 0)$ is globally Lispchitz and that there exists $c>0$ and $q \in[0 ; 1)$ such that, for all $\phi \in \mathcal{C}^{n}$ and all $v \in \mathbb{R}^{m}$,

$$
|f(\phi, v)-f(\phi, 0)| \leq c \max \left\{\|\phi\|^{q} ; 1\right\}|v| .
$$

Then system (2) is ISS. 
Proof: By Theorem 1, the origin of the input-free system $\dot{x}(t)=f\left(x_{t}, 0\right)$ is GES. ISS of (2) then follows from [22, Theorem 3.2].

The regularity assumption (3) is fulfilled in particular if $f$ is globally Lipschitz in both its arguments (in which case $q=0$ ). This corollary slightly complements [3], which investigates whether a point-wise dissipation is enough to guarantee ISS (note that this question is still open).

\section{Proof of Theorem 1}

The implications $i) \Rightarrow i i)$ and $i i) \Rightarrow i i i)$ are trivial. In [22, Theorem 2.4], it is stated that iv) guarantees the existence of a globally Lipschitz functional $V_{0}: \mathcal{C}^{n} \rightarrow \mathbb{R}_{\geq 0}$ and $\underline{\kappa}, \bar{\kappa}, \kappa>0$ such that, for all $\phi \in \mathcal{C}^{n}, \underline{\kappa}\|\phi\| \leq V_{0}(\phi) \leq$ $\bar{\kappa}\|\phi\|$ and $D_{\overline{1}}^{+} V_{0}(\phi) \leq-\kappa\|\phi\|$. Hence, the functional $V:=$ $V_{0}^{2}$ satisfies $\underline{\kappa}^{2}\|\phi\|^{2} \leq V(\phi) \leq \bar{\kappa}^{2}\|\phi\|^{2}$ and $D_{[\overline{1}}^{+} V(\phi)=$ $2 V_{0}(\phi) D_{\text {(1) }}^{+} V_{0}(\phi) \leq-\kappa \underline{\kappa}\|\phi\|^{2}$. Thus, $i v$ ) implies $i$ ) (acually, with a coercive LKF). So it is sufficient to prove that $i i i) \Rightarrow$ $i v$ ), namely that the existence of a GES LKF with pointwise dissipation guarantees global exponential stability. To that aim, recall that $i i i)$ means that there exists a functional $V: \mathcal{C}^{n} \rightarrow \mathbb{R}_{\geq 0}$, Lipschitz on bounded sets, and $\underline{k}, \bar{k}, k>0$ such that, for all $\phi \in \mathcal{C}^{n}$,

$$
\begin{gathered}
\underline{k}|\phi(0)|^{2} \leq V(\phi) \leq \bar{k}\|\phi\|^{2} \\
D_{\overline{1}}^{+} V(\phi) \leq-k|\phi(0)|^{2} .
\end{gathered}
$$

Let

$$
W(\phi):=\int_{-\delta}^{0} \max _{s \in[\tau ; 0]}|\phi(s)|^{2} d \tau, \quad \forall \phi \in \mathcal{C}^{n} .
$$

The proof consists in showing that the functional

$$
\mathcal{V}(\phi):=V(\phi)+\varepsilon W(\phi),
$$

with some $\varepsilon>0$, has a history-wise dissipation rate along the solutions of (1). We start by showing the following.

Claim 1 Given any $\varepsilon>0, \mathcal{V}$ is Lipschitz on bounded sets.

Proof: Since $V$ is Lipschitz on bounded sets, it is sufficient to show that $W$ enjoys the same property. First observe that, for all $\tau \in[-\delta ; 0]$ and for all $\phi, \psi \in \mathcal{C}^{n}$,

$$
\left.\left|\max _{s \in[\tau ; 0]}\right| \phi(s)\right|^{2}-\max _{s \in[\tau ; 0]}|\psi(s)|^{2}\left|\leq \max _{s \in[\tau ; 0]}\right||\phi(s)|^{2}-|\psi(s)|^{2} \mid .
$$

To see this, consider first the case when $\max _{s \in[\tau ; 0]}|\phi(s)|^{2} \geq$ $\max _{s \in[\tau ; 0]}|\psi(s)|^{2}$ and let $s^{*} \in[\tau ; 0]$ be such that $\max _{s \in[\tau ; 0]}|\phi(s)|^{2}=\left|\phi\left(s^{*}\right)\right|^{2}$. Then it holds that

$$
\begin{aligned}
\max _{s \in[\tau ; 0]}|\phi(s)|^{2} & -\max _{s \in[\tau ; 0]}|\psi(s)|^{2} \mid \\
& =\left|\phi\left(s^{*}\right)\right|^{2}-\max _{s \in[\tau ; 0]}|\psi(s)|^{2} \\
& \leq\left|\phi\left(s^{*}\right)\right|^{2}-\left|\psi\left(s^{*}\right)\right|^{2} \\
& \leq \max _{s \in[\tau ; 0]}\left(|\phi(s)|^{2}-|\psi(s)|^{2}\right) \\
& \leq\left.\max _{s \in[\tau ; 0]}|| \phi(s)\right|^{2}-|\psi(s)|^{2} \mid
\end{aligned}
$$

The case when $\max _{s \in[\tau ; 0]}|\phi(s)|^{2} \leq \max _{s \in[\tau ; 0]}|\psi(s)|^{2}$ can be addressed in the same way, which establishes (7). Let $\mathcal{X}$ be any bounded set of $\mathcal{C}^{n}$ and assume that $\phi, \psi \in \mathcal{X}$. Since the function $x \mapsto x^{2}$ is locally Lipschitz, there exists $\ell>0$ such that $\left.|| \phi(s)\right|^{2}-|\psi(s)|^{2}|\leq \ell||\phi(s)|-|\psi(s)| \mid \leq$ $\ell|\phi(s)-\psi(s)|$. It follows from (7) that, for all $\tau \in[-\delta ; 0]$ and all $\phi, \psi \in \mathcal{X}$,

$$
\begin{aligned}
\max _{s \in[\tau ; 0]}|\phi(s)|^{2}-\max _{s \in[\tau ; 0]}|\psi(s)|^{2} \mid & \leq \ell \max _{s \in[\tau ; 0]}|\phi(s)-\psi(s)| \\
& \leq \ell\|\phi-\psi\| .
\end{aligned}
$$

Thus, in view of 6 , it holds for all $\phi, \psi \in \mathcal{X}$ that

$$
\begin{aligned}
\mid W(\phi) & -W(\psi) \mid \\
& =\left.\left|\int_{-\delta}^{0} \max _{s \in[\tau ; 0]}\right| \phi(s)\right|^{2} d \tau-\int_{-\delta}^{0} \max _{s \in[\tau ; 0]}|\psi(s)|^{2} d \tau \mid \\
& \leq\left.\int_{-\delta}^{0}\left|\max _{s \in[\tau ; 0]}\right| \phi(s)\right|^{2}-\max _{s \in[\tau ; 0]}|\psi(s)|^{2} \mid d \tau \\
& \leq \ell \delta\|\phi-\psi\|,
\end{aligned}
$$

meaning that $W$ is Lipschitz on bounded sets.

Using (4) and (6), we also have the following.

Claim 2 Given any $\varepsilon>0$ it holds that

$$
\underline{k}|\phi(0)|^{2} \leq \mathcal{V}(\phi) \leq(\bar{k}+\varepsilon \delta)\|\phi\|^{2}, \quad \forall \phi \in \mathcal{C}^{n} .
$$

We now proceed to computing the Dini derivative of $\mathcal{V}$ along the solutions of (1). To that aim, consider the solution $t \mapsto x_{t}$ of (1) starting from any $x_{0} \in \mathcal{C}^{n}$ at $t=0$. Note that, since $f$ is globally Lipschitz, this solution exists at all times $t \geq 0$ and is unique [5]. Hence, for all $t \geq 0$, it holds that

$$
W\left(x_{t}\right)=\int_{-\delta}^{0} \max _{s \in[\tau ; 0]}|x(t+s)|^{2} d \tau .
$$

Operating successively the changes of variables $s \leftarrow t+s$ and $\tau \leftarrow \tau+t$, it follows that

$$
W\left(x_{t}\right)=\int_{t-\delta}^{t} \max _{s \in[\tau ; t]}|x(s)|^{2} d \tau .
$$

The following statement constitutes the key idea of the paper: adding $W$ to the GES LKF $V$ with point-wise dissipation allows to obtain a history-wise dissipation term.

Claim 3 For all $t \geq 0$, it holds that

$$
D_{\text {囬 }}^{+} W\left(x_{t}\right) \leq|x(t)|^{2}-\left\|x_{t}\right\|^{2}+2 \delta|x(t)|\left|f\left(x_{t}\right)\right| .
$$

Proof: If $\tau \mapsto \max _{s \in[\tau ; t]}|x(s)|^{2}$ were differentiable, then the derivative of $W$ could be easily computed using Leibniz integral rule. Unfortunately, this may not be the case. This is why the proof of this claim is a bit technical. In order to lighten the notation, define:

$$
m(\tau, t):=\max _{s \in[\tau ; t]}|x(s)|^{2}, \quad \forall t \geq \tau \geq 0 .
$$

Then the function $m$ satisfies the following: 
P1 $m(\tau, t) \geq 0$ for all $t \geq \tau \geq 0$

P2 $\tau \mapsto m(\tau, t)$ is non-increasing on $[0 ; t]$

P3 $t \mapsto m(\tau, t)$ is non-decreasing on $[\tau ;+\infty)$.

P4 $m(\tau, t)=\max \{m(\tau, s) ; m(s, t)\}$ for all $t \geq s \geq \tau \geq 0$.

With this notation, $W\left(x_{t}\right)=\int_{t-\delta}^{t} m(\tau, t) d \tau$, hence:

$D_{\text {囬 }}^{+} W\left(x_{t}\right)=\limsup _{h \rightarrow 0^{+}} \frac{1}{h}\left(\int_{t+h-\delta}^{t+h} m(\tau, t+h) d \tau-\int_{t-\delta}^{t} m(\tau, t) d \tau\right)$.

Let

$$
I(t):=\limsup _{h \rightarrow 0^{+}} \frac{1}{h} \int_{t+h-\delta}^{t+h}(m(\tau, t+h)-m(\min \{\tau ; t\}, t)) d \tau .
$$

Then it holds that

$$
\begin{aligned}
& D_{\text {志 }}^{+} W\left(x_{t}\right) \leq I(t) \\
& +\limsup _{h \rightarrow 0^{+}} \frac{1}{h}\left(\int_{t+h-\delta}^{t+h} m(\min \{\tau ; t\}, t) d \tau-\int_{t-\delta}^{t} m(\tau, t) d \tau\right) .
\end{aligned}
$$

Notice that this latter term reads

$$
\begin{aligned}
& \limsup _{h \rightarrow 0^{+}} \frac{1}{h}\left(\int_{t+h-\delta}^{t+h} m(\min \{\tau ; t\}, t) d \tau-\int_{t-\delta}^{t} m(\tau, t) d \tau\right) \\
& =\limsup _{h \rightarrow 0^{+}} \frac{1}{h}\left(\int_{t}^{t+h} m(t, t) d \tau+\int_{t+h-\delta}^{t} m(\tau, t) d \tau-\int_{t-\delta}^{t} m(\tau, t) d \tau\right) \\
& =\limsup _{h \rightarrow 0^{+}} \frac{1}{h}\left(\int_{t}^{t+h} m(t, t) d \tau-\int_{t-\delta}^{t+h-\delta} m(\tau, t) d \tau\right) \\
& =m(t, t)-m(t-\delta, t) \\
& =|x(t)|^{2}-\left\|x_{t}\right\|^{2},
\end{aligned}
$$

where the last equality comes from (10). It follows that

$$
D_{\text {苗 }}^{+} W\left(x_{t}\right) \leq I(t)+|x(t)|^{2}-\left\|x_{t}\right\|^{2} .
$$

So all we need to show is that $I(t) \leq 2 \delta|x(t)|\left|f\left(x_{t}\right)\right|$. In view of (11), $I(t)$ can be written as

$$
I(t)=\limsup _{h \rightarrow 0^{+}} \frac{J(h, t)}{h},
$$

where

$$
\begin{aligned}
J(h, t):= & \int_{t+h-\delta}^{t+h}(m(\tau, t+h)-m(\min \{\tau ; t\}, t)) d \tau \\
= & \int_{t+h-\delta}^{t}(m(\tau, t+h)-m(\min \{\tau ; t\}, t)) d \tau \\
& +\int_{t}^{t+h}(m(\tau, t+h)-m(\min \{\tau ; t\}, t)) d \tau \\
= & \int_{t+h-\delta}^{t}(m(\tau, t+h)-m(\tau, t)) d \tau \\
& +\int_{t}^{t+h}(m(\tau, t+h)-m(t, t)) d \tau .
\end{aligned}
$$

Since $m(t, t)=|x(t)|^{2}$, we get that

$$
\begin{aligned}
J(h, t)= & \int_{t+h-\delta}^{t}(m(\tau, t+h)-m(\tau, t)) d \tau \\
& +\int_{t}^{t+h}\left(m(\tau, t+h)-|x(t)|^{2}\right) d \tau \\
= & \int_{t-\delta}^{t}(m(\tau, t+h)-m(\tau, t)) d \tau \\
& -\int_{t-\delta}^{t+h-\delta}(m(\tau, t+h)-m(\tau, t)) d \tau \\
& +\int_{t}^{t+h}\left(m(\tau, t+h)-|x(t)|^{2}\right) d \tau .
\end{aligned}
$$

Observing that the second integral of this expression is nonnegative (due to $\mathrm{P} 3$ ), we get that

$$
\begin{aligned}
J(h, t) \leq & \int_{t-\delta}^{t}(m(\tau, t+h)-m(\tau, t)) d \tau \\
& +\int_{t}^{t+h}\left(m(\tau, t+h)-|x(t)|^{2}\right) d \tau .
\end{aligned}
$$

It follows from (13) that $I(t) \leq I_{1}(t)+I_{2}(t)$, where

$$
\begin{aligned}
& I_{1}(t):=\limsup _{h \rightarrow 0^{+}} \frac{1}{h} \int_{t-\delta}^{t}(m(\tau, t+h)-m(\tau, t)) d \tau \\
& I_{2}(t):=\limsup _{h \rightarrow 0^{+}} \frac{1}{h} \int_{t}^{t+h}\left(m(\tau, t+h)-|x(t)|^{2}\right) d \tau .
\end{aligned}
$$

We start by computing $I_{2}(t)$ : using $\mathrm{P} 2$, it holds that

$$
\begin{aligned}
I_{2}(t) & \leq \limsup _{h \rightarrow 0^{+}} \frac{1}{h} \int_{t}^{t+h}\left(m(t, t+h)-|x(t)|^{2}\right) d \tau \\
& \leq \limsup _{h \rightarrow 0^{+}} m(t, t+h)-|x(t)|^{2}=0,
\end{aligned}
$$

using (10) and the continuity of $s \mapsto|x(s)|$. Thus, $I(t) \leq$ $I_{1}(t)$. Moreover, using P4, it holds for all $t \geq \tau$ that $m(\tau, t+$ $h)=\max \{m(\tau, t) ; m(t, t+h)\}$. It follows that

$$
\begin{aligned}
I_{1}(t) & =\limsup _{h \rightarrow 0^{+}} \frac{1}{h} \int_{t-\delta}^{t}(m(\tau, t+h)-m(\tau, t)) d \tau \\
& =\limsup _{h \rightarrow 0^{+}} \frac{1}{h} \int_{t-\delta}^{t} \max \{0 ; m(t, t+h)-m(\tau, t)\} d \tau \\
& \leq \limsup _{h \rightarrow 0^{+}} \frac{\delta}{h} \max \left\{0 ; m(t, t+h)-|x(t)|^{2}\right\},
\end{aligned}
$$

since $m(\tau, t)=\max _{s \in[\tau ; t]}|x(s)|^{2} \geq|x(t)|^{2}$. Furthermore, for all $s \geq t, x(s)=x(t)+\int_{t}^{s} f\left(x_{\theta}\right) d \theta$. It follows that

$$
\begin{aligned}
& m(t, t+h)=\max _{s \in[t ; t+h]} x(s)^{T} x(s) \\
& =\max _{s \in[t ; t+h]}|x(t)|^{2}+2 x(t)^{T} \int_{t}^{s} f\left(x_{\theta}\right) d \theta+\left|\int_{t}^{s} f\left(x_{\theta}\right) d \theta\right|^{2} \\
& \leq|x(t)|^{2}+2|x(t)| \int_{t}^{t+h}\left|f\left(x_{\theta}\right)\right| d \theta+\left(\int_{t}^{t+h}\left|f\left(x_{\theta}\right)\right| d \theta\right)^{2} .
\end{aligned}
$$

Recalling that $m(t, t)=|x(t)|^{2}$, we obtain:

$I_{1}(t) \leq \limsup _{h \rightarrow 0^{+}} \frac{\delta}{h}\left(2|x(t)| \int_{t}^{t+h}\left|f\left(x_{\theta}\right)\right| d \theta+\left(\int_{t}^{t+h}\left|f\left(x_{\theta}\right)\right| d \theta\right)^{2}\right)$. 
Now, observe that (5) ensures in particular that $D_{[1}^{+} V\left(x_{t}\right) \leq$ 0 . This, combined with (4), guarantees that $|x(t)| \leq$ $\sqrt{\frac{\bar{k}}{\underline{k}}}\left\|x_{0}\right\|$, which in turn implies that $\left\|x_{t}\right\| \leq \sqrt{\frac{\bar{k}}{\underline{k}}}\left\|x_{0}\right\|$. Since $f$ is globally Lipschitz and $f(0)=0$, there exists a positive constant $\ell_{f}$ such that $\left|f\left(x_{t}\right)\right| \leq \ell_{f}\left\|x_{0}\right\|$. Consequently:

$$
\begin{aligned}
& I(t) \leq I_{1}(t) \\
& \leq 2 \delta|x(t)| \limsup _{h \rightarrow 0^{+}} \frac{1}{h} \int_{t}^{t+h}\left|f\left(x_{\theta}\right)\right| d \theta+\limsup _{h \rightarrow 0^{+}} \frac{h^{2} \ell_{f}^{2}\left\|x_{0}\right\|^{2}}{h} \\
& \leq 2 \delta|x(t)|\left|f\left(x_{t}\right)\right| .
\end{aligned}
$$

The claim then follows from 12 .

We can now conclude the proof of Theorem 11. Since $\left|f\left(x_{t}\right)\right| \leq \ell_{f}\left\|x_{t}\right\|$ for some $\ell_{f}>0$, it follows from Claim 3 that, for all $t \geq 0$,

$$
D_{\text {直 }}^{+} W\left(x_{t}\right) \leq|x(t)|^{2}-\left\|x_{t}\right\|^{2}+2 \delta \ell_{f}|x(t)|\left\|x_{t}\right\| .
$$

Now, recall that $\mathcal{V}(\phi):=V(\phi)+\varepsilon W(\phi)$, with $\varepsilon>0$ to be chosen. (5) and (14) ensure that, for any $\lambda>0$,

$$
\begin{gathered}
D_{\text {1] }}^{+} \mathcal{V}\left(x_{t}\right) \leq-k|x(t)|^{2}+\varepsilon|x(t)|^{2}-\varepsilon\left\|x_{t}\right\|^{2}+2 \varepsilon \delta \ell_{f}|x(t)|\left\|x_{t}\right\| \\
\leq-(k-\varepsilon)|x(t)|^{2}-\varepsilon\left\|x_{t}\right\|^{2}+\varepsilon \delta \ell_{f}\left(\lambda|x(t)|^{2}+\frac{\left\|x_{t}\right\|^{2}}{\lambda}\right) \\
\leq-\left(k-\varepsilon-\varepsilon \delta \ell_{f} \lambda\right)|x(t)|^{2}-\varepsilon\left(1-\frac{\delta \ell_{f}}{\lambda}\right)\left\|x_{t}\right\|^{2} .
\end{gathered}
$$

Pick $\lambda=2 \delta \ell_{f}$, then

$$
D_{\text {菑 }}^{+} \mathcal{V}\left(x_{t}\right) \leq-\left(k-\varepsilon-2 \varepsilon \delta^{2} \ell_{f}^{2}\right)|x(t)|^{2}-\frac{\varepsilon}{2}\left\|x_{t}\right\|^{2} .
$$

Thus, by picking $\varepsilon:=k /\left(1+2 \delta^{2} \ell_{f}^{2}\right)$, we finally obtain that

$$
D_{\text {直 }}^{+} \mathcal{V}\left(x_{t}\right) \leq-\frac{\varepsilon}{2}\left\|x_{t}\right\|^{2}, \quad \forall t \geq 0 .
$$

From this, a strategy to conclude GES is to use the bounds on $\mathcal{V}$ from Claim 2 to show the exponential decay of $\mathcal{V}\left(x_{t}\right)$ with the help of a comparison lemma by taking into account that $V$ is locally Lipschitz (thus the problem of the absolute continuity is overcome, see [16]), and that the function $t \mapsto$ $W\left(x_{t}\right)$ is locally absolutely continuous. An alternative is to directly integrate $[15$ to get from Claim 2 that

$$
\int_{0}^{\infty}\left\|x_{t}\right\|^{2} d t \leq \frac{2}{\varepsilon} V\left(x_{0}\right) \leq \frac{2 \bar{k}}{\varepsilon}\left\|x_{0}\right\|^{2}, \quad \forall x_{0} \in \mathcal{C}^{n},
$$

and GES follows from the integral criterion proposed in [6].

\section{EXAMPLE}

In [13], a model of two interconnected neuronal populations was proposed to explain a possible mechanism for parkinsonian brain oscillations onset. This model reads:

$$
\begin{aligned}
& \tau_{1} \dot{x}_{1}=-x_{1}+S_{1}\left(c_{11} x_{1}\left(t-\delta_{11}\right)-c_{12} x_{2}\left(t-\delta_{12}\right)+\mu\right) \\
& \tau_{2} \dot{x}_{2}=-x_{2}+S_{2}\left(c_{21} x_{1}\left(t-\delta_{21}\right)-c_{22} x_{2}\left(t-\delta_{22}\right)\right)
\end{aligned}
$$

where the argument $t$ of the non-delayed variables have been omitted. In this model, $x_{1}(t)$ and $x_{2}(t)$ represent the neuronal activity of populations 1 and 2. $\tau_{1}, \tau_{2}>0$ are time constants. $S_{1}, S_{2}: \mathbb{R} \rightarrow \mathbb{R}$ are Lipschitz function (with no loss of generality, we assume their Lipschitz constant is $1)$. For each $i, j \in\{1,2\}, c_{i j} \geq 0$ represents the synaptic strength from population $j$ to population $i$ and the delays $\delta_{i j} \geq 0$ take into account the finite velocity of action potentials propagation from population $j$ to population $i$. $\mu(t) \in \mathbb{R}$ represents an artificial stimulation signal that can be introduced through implanted electrodes in order to disrupt pathological oscillations.

With appropriate parameter values, this system exhibits sustained pathological oscillations as a result of instability caused by strong synaptic connectivity between the two populations [13], [15]. This model has been studied in [4], using linearization around equilibrium, to show that a proportional feedback strategy from Population 1 to itself is able to locally stabilize the system. This was confirmed in a spatio-temporal extension of this model [1]. The following result states that this feedback globally exponentially stabilizes the system.

Proposition 1 For each $i, j \in\{1,2\}$, let $c_{i j}, \delta_{i j} \geq 0$ and $\tau_{i}>0$. Assume that functions $S_{i}$ are globally Lipschitz with Lipschitz constant 1 , non-decreasing, and such that $S_{i}(0)=$ 0 . Then there exists $\theta^{*} \geq 0$ such that, for all $\theta \geq \theta^{*}$, the system (16) in closed loop with $\mu(t)=-\theta x_{1}(t)$ is GES.

The proof consists in finding a GES LKF with point-wise dissipation. For this particular example, one could find a strict LKF (by putting exponential terms in the kernels of (18) below), but at the price of more involved computations. Note that if additive disturbances act in the dynamics (16, whether inside or outside the functions $S_{i}$, ISS can easily be derived based on Corollary 1 .

Proof: For any $\theta \geq 0$, the closed-loop dynamics reads:

$$
\begin{aligned}
& \tau_{1} \dot{x}_{1}=-x_{1}+S_{1}\left(c_{11} x_{1}\left(t-\delta_{11}\right)-c_{12} x_{2}\left(t-\delta_{12}\right)-\theta x_{1}\right) \\
& \tau_{2} \dot{x}_{2}=-x_{2}+S_{2}\left(c_{21} x_{1}\left(t-\delta_{21}\right)-c_{22} x_{2}\left(t-\delta_{22}\right)\right) .
\end{aligned}
$$

Let $V(\phi)=V_{1}\left(\phi_{1}\right)+\rho V_{2}\left(\phi_{2}\right)$ where, for each $j \in\{1,2\}$,

$$
V_{j}(\phi)=\frac{1}{2}\left(\tau_{j} \phi(0)^{2}+\sum_{i=1}^{2} \int_{-\delta_{i j}}^{0} \lambda_{i j} \phi(s)^{2} d s\right),
$$

with $\rho>0$ and $\lambda_{i j}>0$ to be chosen. $V$ is Lipschitz on bounded sets. Moreover, it satisfies bounds of the form (4) by setting $\bar{k}=\tau_{1}+\rho \tau_{2}+\lambda_{11} \delta_{11}+\lambda_{21} \delta_{21}+\rho \lambda_{12} \delta_{12}+\rho \lambda_{22} \delta_{22}$ and $\underline{k}=\min \left\{\tau_{1}, \tau_{2}\right\} \min \{1, \rho\} / 2$. Moreover, for all $\phi \in \mathcal{C}^{2}$,

$$
\begin{aligned}
& D_{[17}^{+} V=-\phi_{1}(0)^{2}-\rho \phi_{2}(0)^{2}+\phi_{1}(0) S_{1}\left(c_{11} \phi_{1}\left(-\delta_{11}\right)-c_{12} \phi_{2}\left(-\delta_{12}\right)\right. \\
& \left.-\theta \phi_{1}(0)\right)+\rho \phi_{2}(0) S_{2}\left(c_{21} \phi_{1}\left(-\delta_{21}\right)-c_{22} \phi_{2}\left(-\delta_{22}\right)\right) \\
& +\sum_{i=1}^{2} \frac{\lambda_{i 1}}{2}\left(\phi_{1}(0)^{2}-\phi_{1}\left(-\delta_{i 1}\right)^{2}\right)+\sum_{i=1}^{2} \frac{\rho \lambda_{i 2}}{2}\left(\phi_{2}(0)^{2}-\phi_{2}\left(-\delta_{i 2}\right)^{2}\right) .
\end{aligned}
$$

Since each $S_{i}$ is globally Lipschitz with Lipschitz constant 1 , nondecreasing, and satisfies $S_{i}(0)=0$, it holds that $\left|S_{i}(s)\right| \leq|s|$ and $S_{i}(s) s \geq 0$ for all $s \in \mathbb{R}$. Based on this, the examination of two cases, whether or not the argument of $S_{1}$ dominates the control signal $-\theta \phi_{1}(0)$, 
shows that $\phi_{1}(0) S_{1}\left(c_{11} \phi_{1}\left(-\delta_{11}\right)-c_{12} \phi_{2}\left(-\delta_{12}\right)-\theta \phi_{1}(0)\right) \leq$ $\frac{4}{\theta}\left(c_{11}^{2} x_{1}\left(t-\delta_{11}\right)^{2}+c_{12}^{2} x_{2}\left(t-\delta_{12}\right)^{2}\right)$. In the same way,

$$
\begin{aligned}
& \phi_{2}(0) S_{2}\left(c_{21} \phi_{1}\left(-\delta_{21}-c_{22} \phi_{2}\left(-\delta_{22}\right)\right) \leq\right. \\
& \frac{c_{21}}{2}\left(\xi_{1} \phi_{2}(0)^{2}+\frac{\phi_{1}\left(-\delta_{21}\right)^{2}}{\xi_{1}}\right)+\frac{c_{22}}{2}\left(\xi_{2} \phi_{2}(0)^{2}+\frac{\phi_{2}\left(-\delta_{22}\right)^{2}}{\xi_{2}}\right) .
\end{aligned}
$$

for any $\xi_{1} ; \xi_{2}>0$. Putting it all together, it follows that

$$
\begin{aligned}
& D_{117}^{+} V(\phi) \leq-\phi_{1}(0)^{2}-\rho \phi_{2}(0)^{2}+\frac{4}{\theta}\left(c_{11}^{2} \phi_{1}\left(-\delta_{11}\right)^{2}\right. \\
& \left.+c_{12}^{2} \phi_{2}\left(-\delta_{12}\right)^{2}\right)+\frac{\rho c_{21}}{2}\left(\xi_{1} \phi_{2}(0)^{2}+\frac{1}{\xi_{1}} \phi_{1}\left(-\delta_{21}\right)^{2}\right) \\
& +\frac{\rho c_{22}}{2}\left(\xi_{2} \phi_{2}(0)^{2}+\frac{1}{\xi_{2}} \phi_{2}\left(-\delta_{22}\right)^{2}\right) \\
& +\frac{1}{2} \sum_{i=1}^{2} \lambda_{i 1}\left(\phi_{1}(0)^{2}-\phi_{1}\left(-\delta_{i 1}\right)^{2}\right)+\rho \lambda_{i 2}\left(\phi_{2}(0)^{2}-\phi_{2}\left(-\delta_{i 2}\right)^{2}\right) .
\end{aligned}
$$

Regrouping terms, it follows that $D_{[17,}^{+} V(\phi) \leq$ $-\frac{1-c_{22}}{2}|\phi(0)|^{2}$, provided that:
(a) : $\lambda_{11}+\lambda_{21}<2$,
(b) : $\frac{\rho c_{21}}{\xi_{1}} \leq \lambda_{21}$
(c) : $c_{21} \xi_{1}+c_{22} \xi_{2}+\lambda_{12}+\lambda_{22}<2$,
(d) : $\frac{8}{\theta \rho} c_{12}^{2} \leq \lambda_{12}$,
(e) : $\frac{8}{\theta} c_{11}^{2} \leq \lambda_{11}$,
(f) : $\frac{c_{22}}{\xi_{2}} \leq \lambda_{22}$.

By setting $\lambda_{22}=\frac{c_{22}}{\xi_{2}},(f)$ is fulfilled and $(c)$ reads $c_{21} \xi_{1}+c_{22}\left(\xi_{2}+1 / \xi_{2}\right)+\lambda_{12}<2$. For positive arguments, the function $x \mapsto x+1 / x$ has a minimum for $x=1$, so we set $\xi_{2}=1$. By setting $\lambda_{12}=\frac{1-c_{22}}{2}$ and $\xi_{1}=\frac{\lambda_{12}}{c_{21}}=\frac{1-c_{22}}{2 c_{21}}$, (c) is fulfilled provided that $c_{22}<1$, which we have by assumption. The choice $\rho=\frac{8}{\theta \lambda_{12}} c_{12}^{2}=\frac{16 c_{12}^{2}}{\theta\left(1-c_{22}\right)}$ validates $(d)$. Moreover, by letting $\lambda_{11}=\frac{8}{\theta} c_{11}^{2}$ and $\lambda_{21}=\frac{\rho c_{21}}{\xi_{1}}=$ $\frac{2 \rho c_{21}^{2}}{1-c_{22}},(e)$ and $(b)$ are fulfilled and $(a)$ reads $\frac{8}{\theta} c_{11}^{2}+\frac{2 \rho c_{21}^{2}}{1-c_{22}}<$ 2. Replacing $\rho$ by its value, possible choices of $\theta$ are:

$$
\theta \geq \theta^{*}:=8\left(c_{11}^{2}+\frac{4 c_{21}^{2} c_{12}^{2}}{\left(1-c_{22}\right)^{2}}\right) .
$$

By Theorem 1. 177) is GES for these values.

\section{CONCLUSIONS AND PERSPECTIVES}

In this paper, we have shown that GES can be established with a functional that dissipates only point-wisely, if the dynamics are globally Lipschitz. We have quickly evoked some consequences in terms of robustness to exogenous inputs and illustrated its applicability through an example taken from neuroscience literature.

The restriction to globally Lipschitz dynamics is of course the main drawback of the approach: further work is needed to investigate whether the proposed LKF construction can be extended to merely locally Lipschitz systems. Also, the LKF considered here have quadratic bounds: deeper investigations are required to check whether bounds in the power $p \geq 1$ can also be employed. The proof presented here suggests that this extension is feasible for $p>1$, but linear bounds $(p=1)$ seem less straightforward. This is a pity as converse results for GES do make use of linear bounds [18] and such an extension would probably allow to construct a globally Lipschitz LKF with history-wise dissipation (which would be expected in view of the strong regularity requirements imposed on the fector field). We also hope that the proposed LKF construction will be of some help to solve the conjecture posed in [3] about ISS based on point-wise dissipation.

\section{ACKNOWLEDGMENT}

The authors wish to thank M. Malisoff, P. Mason, and M. Sigalotti for fruitful discussions about this question.

\section{REFERENCES}

[1] A. Chaillet, G. Detorakis, S. Palfi, and S. Senova. Robust stabilization of delayed neural fields with partial measurement and actuation. Automatica, 83:262-274, Sep. 2017.

[2] A. Chaillet and P. Pepe. Integral input-to-state stability of delay systems based on Lyapunov-Krasovskii functionals with point-wise dissipation rate. In IEEE Conf. on Decision and Control, 2018.

[3] A. Chaillet, P. Pepe, P. Mason, and Y. Chitour. Is a point-wise dissipation rate enough to show ISS for time-delay systems? In IFAC World Congress, Toulouse, France, July 2017.

[4] I. Haidar, W. Pasillas-Lépine, A. Chaillet, E. Panteley, S. Palfi, and S. Senova. A firing-rate regulation strategy for closed-loop deep brain stimulation. Biological Cybernetics, 110(1):55-71, 2016.

[5] J.K. Hale. Theory of functional differential equations. Applied mathematical sciences, pages 1-376, 1977.

[6] A. Ichikawa. Equivalence of $L_{p}$ stability and exponential stability for a class of nonlinear semigroups. Nonlinear analysis, theory, methods, and applications, 8(7):805-815, 1984.

[7] H. Ito, P. Pepe, and Z.P. Jiang. A small-gain condition for iISS of interconnected retarded systems based on Lyapunov-Krasovskii functionals. Automatica, 46(10):1646-1656, October 2010.

[8] I. Karafyllis and Z.P. Jiang. Stability and stabilization of nonlinear systems. Comm. and Contr. Eng. Series. Springer-Verlag, 2011.

[9] I. Karafyllis, P. Pepe, and Z.P. Jiang. Input-to-Output Stability for systems described by retarded functional differential equations. European Journal of Control, 14(6):539-555, 2008.

[10] N.N. Krasovskii. Problems of the theory of stability of Motion. Stanford Univ. Press, 1963.

[11] F. Mazenc, H. Ito, and P. Pepe. Construction of Lyapunov functionals for coupled differential and continuous time difference equations. In Proc. IEEE Conf. on Dec. and Control, pages 2245 - 2250, 2013.

[12] A. Mironchenko and F. Wirth. Global converse Lyapunov theorems for infinite-dimensional systems. In 10th IFAC Symposium on Nonlinear Control Systems (NOLCOS), pages 909-914, 2016.

[13] A.J. Nevado-Holgado, J.R. Terry, and R. Bogacz. Conditions for the generation of beta oscillations in the subthalamic nucleus-globus pallidus network. J. of Neuroscience, 30(37):12340-12352, 2010.

[14] S.I. Niculescu. Delay effects on stability: A robust control approach, volume 269. Springer, 2001.

[15] W. Pasillas-Lépine. Delay-induced oscillations in Wilson and Cowan's model: An analysis of the subthalamo-pallidal feedback loop in healthy and parkinsonian subjects. Biolog. Cyb., 107(3):289-308, 2013.

[16] P. Pepe. The problem of the absolute continuity for LyapunovKrasovskii functionals. IEEE Trans. Autom. Control, 52(5):953-957, 2007.

[17] P. Pepe and Z.P. Jiang. A Lyapunov-Krasovskii methodology for ISS and iISS of time-delay systems. Systems \& Control Letters, 55(12):1006-1014, 2006.

[18] P. Pepe and I. Karafyllis. Converse Lyapunov-Krasovskii theorems for systems described by neutral functional differential equations in Hale's form. International Journal of Control, 86(2):232-243, February 2013.

[19] E.D. Sontag. Smooth stabilization implies coprime factorization. IEEE Trans. Autom. Control, 34(4):435-443, 1989.

[20] E.D. Sontag. Input to state stability: Basic concepts and results. Lecture Notes in Mathematics. Springer-Verlag, Berlin, 2008.

[21] A.R. Teel. Connections between Razumikhin-type theorems and the ISS nonlinear small gain theorem. IEEE Trans. Autom. Control, 43(7):960-964, 1998.

[22] N. Yeganefar, P. Pepe, and M. Dambrine. Input-to-State Stability of time-delay systems: a link with exponential stability. IEEE Trans. Automatic Control, 53(6):1526-1531, 2008. 